\title{
Distal Protein Sequences Can Affect the Function of a Nuclear Localization Signal
}

\author{
MIN GAO† AND DAVID M. KNIPE* \\ Department of Microbiology and Molecular Genetics, Harvard Medical School, \\ 200 Longwood Avenue, Boston, Massachusetts 02115
}

Received 30 July 1991/Accepted 18 December 1991

\begin{abstract}
The major DNA-binding protein, or infected-cell protein 8 (ICP8), encoded by herpes simplex virus can localize to the cell nucleus independently of other viral proteins. To define the nuclear localization signals within ICP8, we performed several forms of mutagenesis on the cloned ICP8 gene. Deletion analysis of the ICP8 gene showed that several portions of ICP8 are involved in its nuclear localization. To determine whether these regions were independent localization signals, we introduced various portions of the ICP8 gene into a series of cassette plasmids which allowed expression of fusion proteins containing pyruvate kinase, normally a cytoplasmic protein, fused to various portions of ICP8. These results showed that the carboxyl-terminal 28 residues are the only portion of ICP8 capable of targeting protein kinase into the nucleus. However, inclusion of certain additional regions of ICP8 into the fusion protein led to an inhibition of nuclear localization. Therefore, the carboxyl-terminal 28 residues of ICP8 can act independently as a nuclear localization signal, but certain conformational constraints or folding or assembly requirements in the remainder of the protein can afiect the nuclear localization of the protein. Our results demonstrate that sequences distant from a nuclear localization signal can affect its ability to function. A set of fusion vectors has been isolated which should be of general use for making $5^{\prime}$ or $3^{\prime}$ fusions in any reading frame to rapidly map localization signals.
\end{abstract}

Nuclear localization of proteins is believed to occur through at least two mechanisms: small proteins lacking nuclear localization signals may diffuse into the nucleus through nuclear pores, where they are then sequestered by binding to intranuclear components, whereas larger proteins containing nuclear localization signals are actively transported from the cytoplasm to the nucleus $(2,7,27)$. The latter process has been shown to involve two distinct steps in vitro: binding to the nuclear pore and translocation across the nuclear membrane $(31,38)$.

Amino acid sequences responsible for the selective nuclear uptake of many proteins have been identified. These studies involved either construction of deletion mutants to determine which regions of the proteins are not needed for the nuclear localization or the construction of fusion proteins to define which regions of the proteins can direct cytoplasmic proteins such as pyruvate kinase (PK) (20), $\beta$-galactosidase $(17,27,28,43)$, or invertase $(30)$ into the nucleus. The simian virus 40 (SV40) $\mathrm{T}$ antigen contains one of the bestdefined nuclear localization signals, which consists of a highly basic stretch of amino acids (Pro-Lys-Lys-Lys-ArgLys-Val) $(19,20,22)$. When these residues were fused to a cytoplasmic protein, PK, they could direct the fusion protein into the nucleus (20). Similar experiments were also performed to identify the sequences within other nuclear proteins which are sufficient to direct cytoplasmic proteins to the nucleus $(17,26,27,30,43)$. These studies have shown that nuclear localization signals are often located near the termini of the polypeptides, but this likely represents a need to be exposed on the surface to exert their function $(13,25$, $27,43)$. The position of a nuclear localization signal and the

\footnotetext{
* Corresponding author.

$\dagger$ Present address: Department of Virology, Bristol-Myers Squibb Co., Princeton, NJ 08543.
}

context of surrounding residues have also been shown to affect its function $(30,40)$. Additional factors may determine whether a sequence can serve as a nuclear localization signal in that similar stretches of basic amino acids have been found in many nonnuclear cytoplasmic proteins (44). In addition, nonbasic amino acid sequences can also function as nuclear localization signals $(6,17)$. In recent studies, the structures and compositions of some nuclear localization signals have been found to be more complex than that of the SV40 T-antigen signal $(8,15,16)$. Some contain more than one sequence $(15,28,29,33,42)$, and they can either function alone (15) or complement each other to direct the proteins into nucleus $(28,29)$. In addition, studies of the progesterone receptor indicated that the process of nuclear localization of the hormone receptor is involved in the interaction between two protein monomers (16).

Infected-cell protein 8 (ICP8), encoded by herpes simplex virus, consists of 1,196 amino acid residues and is required for viral DNA replication $(4,5,9)$ and late gene expression (11). In vitro, ICP8 binds to DNA nonspecifically, with some preference for single-stranded DNA $(1,23,35)$, and it can lower the melting temperature of poly(dA-dT) helices (34). ICP8 may also affect the levels of expression of viral genes $(11,14)$. Genetic analyses of ICP8 have demonstrated that several regions are required for nuclear localization. $\mathrm{Re}$ moval of residues 326 to 586,17 to 411 , or 1160 to 1196 of ICP8 blocks nuclear localization of the protein $(10,32)$. Amino acid substitution at residue 119 (Ala to Val) also leads to an inhibition in nuclear localization $(18,47)$.

To further define the region(s) of ICP8 which functions as a nuclear localization signal, we developed a set of PK fusion vectors which provided a means to rapidly scan ICP8 sequences for nuclear localization signals. The results demonstrated that the carboxyl-terminal 28 residues of ICP8 can function as a nuclear localization signal but that sequences distant from this signal can affect its function. 
Plasmid name

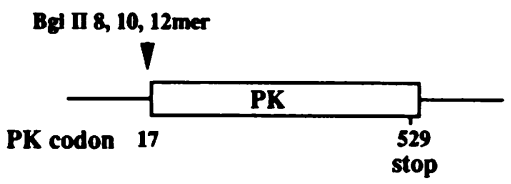

PK 8, 10, 12

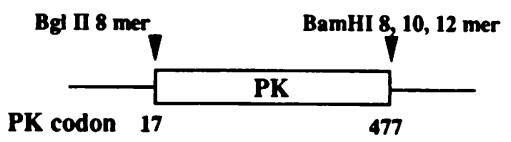

PK8/8, PK8/10, PK8/12

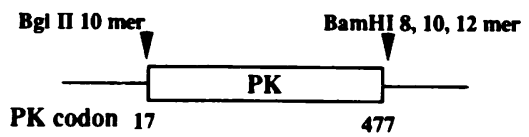

PK10/8, PK10/10, PK10/12

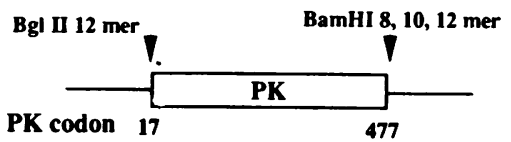

PK12/8, PK12/10, PK12/12

FIG. 1. Structures of PK cassette vectors. Plasmids PK8, PK10, and PK12 have BglII linkers of 8,10 , and $12 \mathrm{bp}$, respectively, inserted immediately prior to codon 17 of the PK gene. Plasmids PK8/8, PK8/10, and PK8/12 have BamHI linkers of 8,10 , and 12 bp, respectively, at the $3^{\prime}$ end of codon 477 of the PK gene. Plasmids PK10/8, PK10/10, PK10/12, PK12/8, PK12/10, and PK12/12 were constructed in an analogous fashion.

\section{MATERIALS AND METHODS}

Plasmids. Plasmid S5-PK10 containing the chicken PK (CPK) gene (provided by A. Smith) was the prototype for the PK cassette constructs. This plasmid was generated by the conversion of the unique PvuII (nucleotides [nt] 129 at codon 17) and ApaI (nt 1893) sites to EcoRI and BglII sites of PK, respectively, and contains the authentic PK gene stop codon at nt 1672 (24). The EcoRI site (nt 129) of S5-PK10 was filled in with Klenow enzyme and converted into a BglII site with 10- or 12-bp linkers. This BglII fragment was ligated into pUC18 to generate the PK cassette plasmids PK10 and PK12 (Fig. 1). The EcoRI site (nt 129) of S5-PK10 was treated with mung bean nuclease and converted into a BglII site with a 10-bp BglII linker. This BglII fragment was ligated into pUC18 to generate the PK cassette plasmid PK8 (Fig. 1). These plasmids were used to construct the hybrid genes expressing the $\mathrm{N}$-terminal portion of ICP8 fused to PK. Plasmids PK8/8, PK8/10, and PK8/12 were constructed by ligation of BamHI linkers of 8, 10, or $12 \mathrm{bp}$ (New England Biolabs, Beverly, Mass.) at the NaeI site (nt 1508) following partial digestion of PK8 with NaeI (Fig. 1). Plasmids PK10/8, PK10/10, PK10/12, PK12/8, PK12/10, and PK12/12 were constructed in an analogous fashion.

The nucleotide numbering system for the ICP8 gene was described previously (9). Plasmid pSV8.3 was derived from pSV8 (9) by deletion of the SacI-BglII fragment containing non-ICP8 coding sequences. Therefore, a 5.5-kbp SmaISacI fragment containing the ICP8 gene was inserted downstream of the SV40 early promoter (9).

Plasmid pn9 was generated in the same way as were pn10 and pn2, described previously (9). After linearization of plasmid pICP8 at nt 3105 by partial digestion with SmaI, a 14-nt $X b a$ I linker containing stop codons in all three reading frames was added. Thus, pn9 encodes the first 832 amino acid residues of ICP8. ICP8 linker insertion mutant plasmids were generated by insertion of a 12-bp BglII linker at different positions of the ICP8 gene after linearization with the blunt-end-cutting enzyme SmaI, NaeI, or PvuII. ICP8 gene deletion mutant plasmids were constructed by ligation of different $B g l \mathrm{II}$ fragments following BglII digestion of two different linker insertion mutant plasmids. Plasmids pLI16, pLI100, pLI200, and pLI447 have BglII linkers of $12 \mathrm{bp}$ inserted after codons $16,100,200$, and 447 , respectively, of the ICP8 gene. The positions of ICP8 linker insertion and deletion mutant constructs are shown in Fig. 2.

The nucleotide sequence of PK was determined by Lonberg and Gilbert (24). The PK gene was subcloned from plasmid S5-PK10, a derivative of L27-PK, into pUC18 by conversion of the EcoRI site to a BglII site (Fig. 1). The ICP8-PK hybrid genes were constructed by fusing different portions of ICP8 with the PK cassettes PK10 and PK12 or their derivatives. The junctions of each fusion protein encoded by these constructs are indicated in Fig. 4 and 6.

PK antibody. Chicken muscle PK was purchased from Sigma. One young adult female New Zealand White rabbit was prebled for normal sera on day 0 and was injected subcutaneously at four sites with $500 \mu \mathrm{g}$ of PK in a total of $1.6 \mathrm{ml}$ of phosphate-buffered saline containing $0.3 \mathrm{ml}$ of complete adjuvant and $0.3 \mathrm{ml}$ of incomplete adjuvant. The rabbit was boosted with the same amount of PK on days 14, 21 , and 45 and was bled on days 30 and 53 . The rabbit was sacrificed, and serum was collected on day 60 .

Indirect immunofluorescence. Vero cells were grown on 12-mm circular glass coverslips as described previously (37) and transfected with 0.5 to $2 \mu \mathrm{g}$ of mutant ICP8 gene or ICP8-PK fusion plasmids. Twenty-four hours after transfection, indirect immunofluorescence was performed as described previously (37). For staining with a single antibody, a 1:50 dilution of anti-ICP8 rabbit polyclonal serum 3-83 (21) or a 1:75 dilution of anti-PK rabbit polyclonal serum and a 1:200 dilution of fluorescein-conjugated goat anti-rabbit immunoglobulin were used. For double staining, cells were incubated with a 1:40 dilution of anti-ICP8 monoclonal antibody 10-E3 (41) and a 1:75 dilution of anti-PK polyclonal serum followed by a 1:100 dilution of rhodamine-conjugated goat anti-mouse antibody and a 1:200 dilution of fluoresceinconjugated anti-rabbit immunoglobulin.

\section{RESULTS}

Several regions of ICP8 affect nuclear localization. Wildtype ICP8 can accumulate in the nucleus of transfected cells independently of other viral proteins (36) (Fig. 3A). Therefore, ICP8 contains within its polypeptide sequence the information necessary for localization into the cell nucleus. Our previous results (10) and the results of others (32) demonstrated that nuclear localization of ICP8 is affected by mutation of at least three regions of the protein: deletion at the extreme carboxyl terminus (10), deletion of residues 325 


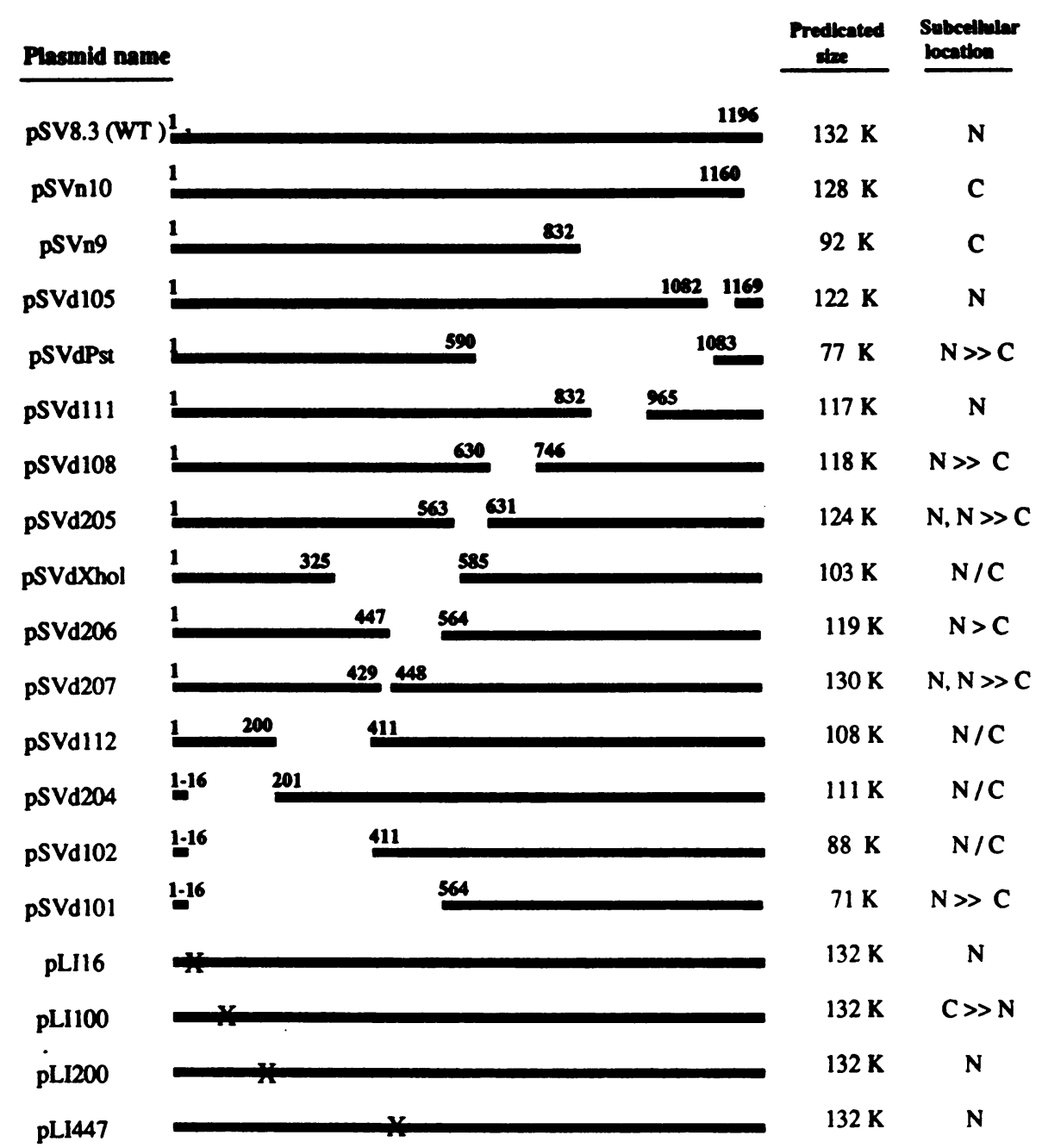

FIG. 2. Structures and subcellular localization of mutant ICP8 molecules. ICP8 gene plasmids containing the indicated mutations were transfected into Vero cells, and the subcellular distribution of the mutant proteins was determined by indirect immunofluorescence staining for ICP8 after $24 \mathrm{~h}$. Solid bars indicate the ICP8 coding sequences present in each of the mutant polypeptides; numbers above the solid bars indicate amino acid residues present in the mutant proteins. X's indicate sites of linker insertions in pL1 plasmids. N, nucleus; C, cytoplasm; N/C, mostly nuclear with some cytoplasmic staining; WT, wild type; K, kilodaltons.

to $411(12,32)$, or amino acid substitution at residue $119(16$, 47) leads to alteration in the nuclear localization of ICP8. The results of these studies, however, were obtained from cells infected with ICP8 mutant viruses. Our preliminary results indicated that the efficiency of ICP8 nuclear localization may be different in cells transfected with mutant plasmids versus cells infected with the viruses containing these mutations. For example, mutant ICP8 molecules were entirely localized in the cytoplasm when cells were infected with $d 102$ and $d$ XhoI viruses $(10,12,32)$, but the nucleus and cytoplasm showed an approximately equal intensity of staining when cells were transfected with plasmids pSVd102 and pSVdXhoI (see below). These findings suggest that other viral protein(s) or events during infection may influence the kinetics or efficiency of nuclear localization of ICP8. Therefore, to define the minimal sequences within ICP8 that determine its nuclear localization, we have examined ICP8 localization in cells transfected with mutant ICP8 gene plasmids.

To define the regions of ICP8 needed for nuclear localization, several ICP8 gene deletion mutant plasmids were constructed. These ICP8 genes were expressed under the control of the SV40 early promoter in transfected Vero cells. The predicted size and observed subcellular location of the mutant ICP8 molecules are shown in Fig. 2. The subcellular distribution of the ICP8 molecules encoded by these constructs was determined by indirect immunofluorescence $24 \mathrm{~h}$ after transfection. We have relied on immunofluorescence techniques to define the subcellular location of the various ICP8 molecules because in some cases, other approaches such as cell fractionation have given misleading results regarding the subcellular location of mutant ICP8 molecules $(12,45)$.

Representative immunofluorescence data are shown in Fig. 3. The results (Fig. 2 and 3) suggested that several regions of ICP8 are required for nuclear localization. The extreme carboxyl terminus of ICP8 is essential for nuclear localization, because loss of residues 1161 to 1196 (SVn10; Fig. 3B) or 833 to 1196 (SVn9; Fig. 2) led to a complete block in nuclear localization. Removal of residues 17 to 200 (SVd204), 17 to 410 (SVd102; Fig. 3C), 200 to 410 (SVd112; Fig. 3D), or 326 to 584 (SVdXhol; Fig. 3E) or a four-amino- 

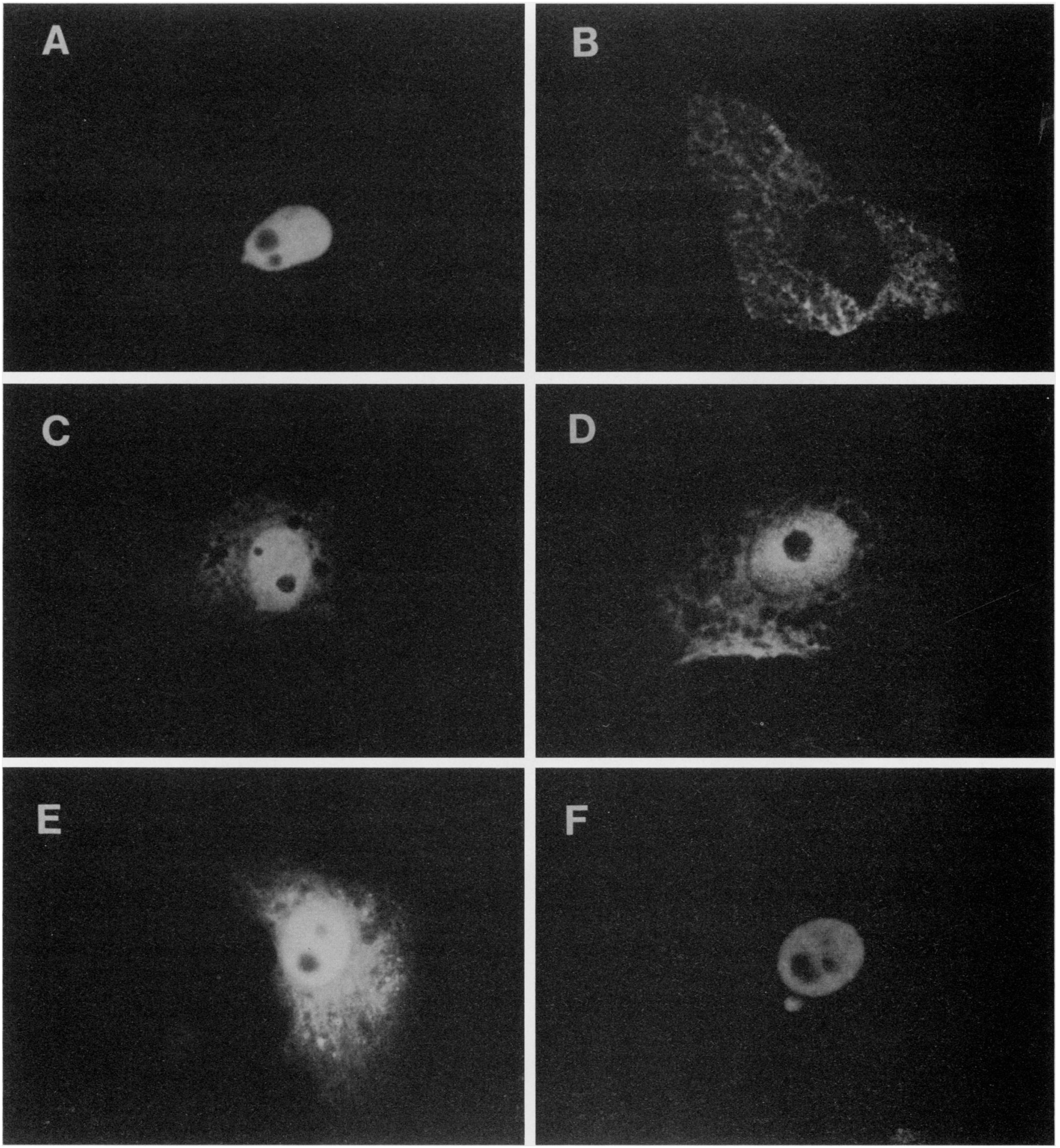

FIG. 3. Immunofluorescence staining for ICP8 in Vero cells transfected with ICP8 plasmids. Twenty-four hours after transfection, Vero cells were fixed, permeabilized, and incubated with either anti-ICP8 monoclonal antibody 10-E3 and rhodamine-conjugated goat anti-mouse immunoglobulin (A and C through F) or anti-ICP8 polyclonal serum 3-83 and fluorescein-conjugated goat anti-rabbit immunoglobulin (B). (A) Wild-type ICP8 plasmid-transfected cells; (B) pSVn10-transfected cells; (C) pSVd102-transfected cells; (D) pSVd112-transfected cells; (E) pSVdXhoI-transfected cells; (F) pSVd105-transfected cells. 
acid insertion after residue 100 (LI100) led to at least a partial block in nuclear localization of ICP8. However, removal of sequences within residues 564 to 1168 (e.g., residues 833 to 964 in SVd111, 1083 to 1168 in SVd105 [Fig. 3F], 591 to 1082 in SVdPst, 631 to 745 in SVd108, or 564 to 630 in SVd205) had little effect on nuclear localization. These results identify two regions as important for nuclear localization: residues 17 to 600 and the carboxyl-terminal 28 residues. The region from residues 17 to 200 overlaps a region previously shown to affect nuclear localization in mutant virus-infected cells (10). Similarly, ICP8 lacking residues 326 to 584 failed to localize to the nucleus $(9,30)$. The last 28 residues of ICP8 seemed to be especially critical for nuclear localization because proteins lacking these residues (SVn9 and SVn10) failed to localize to the nucleus, whereas a protein having residues 1169 to 1196 but lacking residues 1083 to 1168 (SVd105) localized completely to the nucleus. Therefore, multiple regions of ICP8 are required for nuclear localization in transfected cells as well as in infected cells $(10,32)$.

The carboxyl-terminal 28 residues of ICP8 can function as a nuclear localization signal. The deletion analysis described above indicated that the carboxyl-terminal 28 amino acid residues of ICP8 are essential for nuclear localization but that several other regions of the protein also affected its nuclear localization. This approach is limited in that it clearly defines only those regions of ICP8 which are not needed for its nuclear localization. Therefore, to determine which region(s) of ICP8 is sufficient for nuclear localization, we constructed recombinant genes expressing fusion proteins of CPK and various regions of ICP8. PK is normally a cytoplasmic protein $(24,46)$, and PK fusion proteins have been used previously to identify nuclear localization signals (20). In addition to providing an approach for mapping localization signals, the use of fusion proteins would preclude free diffusion of proteins into the cell nucleus because of their size. For example, the small size of the truncated ICP8 molecule expressed by pSVd101 might allow this protein to enter the nucleus by diffusion (12) (Fig. 2). We constructed a set of PK gene plasmids with polylinkers in each of the three reading frames near the $5^{\prime}$ and/or $3^{\prime}$ ends of the PK coding sequences (Fig. 1). This allowed rapid screening of protein sequences within ICP8 which were capable of serving as a nuclear localization signal.

Figure 4 shows the predicted structures of the fusion proteins containing PK fused to various portions of ICP8. Following transfection of the plasmids into Vero cells, the subcellular location of each fusion protein was determined by indirect immunofluorescence with rabbit anti-CPK serum and/or mouse anti-ICP8 monoclonal antibody 10-E3 (Fig. 5). Antibody 10-E3 reacts with the SV8PK9 fusion protein (Fig. $5 F$ ), indicating that it reacts with the carboxyl-terminal 28 residues of ICP8. Double staining of transfected cells with rabbit anti-CPK serum and mouse anti-ICP8 monoclonal antibody 10-E3 allowed us to determine whether the fusion proteins of the hybrid genes were expressed in frame. The other advantage of using double staining is that it allowed us to confirm that the fluorescent staining was detecting intact fusion protein which contained PK determinants as well as the carboxyl-terminal 28 residues of ICP8.

Plasmids PK10 and PK12, containing portions of the SV40 $T$ antigen fused to PK, served as controls for nuclear and cytoplasmic proteins, respectively (Fig. 5A and B). Plasmid SV8PK1, containing the amino-terminal 16 residues of ICP8 fused to PK, also served as a control cytoplasmic protein (12) (Fig. 4). When the entire ICP8 sequence was fused to PK (SV8PK7), the protein localized to the cell nucleus (Fig.
5C). In contrast, while residues 17 to 564 of ICP8 could not direct PK to the nucleus (SV8PK3; Fig. 5D), the carboxylterminal residues 966 to 1196 could do so (SV8PK5; Fig. 5E). Thus, the nuclear localization signal appeared to be at the carboxyl terminus of ICP8. Smaller portions of the carboxyl terminus of ICP8 were fused to PK. Residues 1169 to 1196 of ICP8 were also capable of targeting PK to the nucleus (SV8PK9; Fig. 5F). Thus, this 28-residue region of ICP8 is sufficient to serve as a nuclear localization signal, and this is the only region of ICP8 of those that we tested which was capable of targeting PK to the nucleus.

Sequences distant from a nuclear localization signal can affect its function. The experiments described above demonstrated that the carboxyl-terminal 28 residues of ICP8 could function as a nuclear localization signal when fused to PK, but that these residues were insufficient to direct ICP8 itself into the nucleus when other amino-terminal sequences of the protein were deleted (SVd112, SVdXhoI, SVd204 and LI100 in Fig. 3D and E; Fig. 2). To determine whether aminoterminal sequences of ICP8 would affect the ability of the carboxyl-terminal 28 residues to direct PK into the nucleus, we added some of the amino-terminal regions of ICP8 to the fusion protein SV8PK8. The constructs were designed with an amino-terminal sequence of ICP8 fused to the amino terminus of PK so that the additional ICP8 segments would be less likely to directly affect the folding or availability of the carboxyl-terminal ICP8 nuclear localization signal (Fig. 6). However, ICP8 residues 411 to 1082 (SV8PK11) did inhibit the ability of the carboxyl-terminal 28 residues to direct PK into the nucleus (Fig. 7A). Two other constructs, SV8PK12 and SV8PK13 (Fig. 7B and C), also demonstrated that amino-terminal sequences of ICP8 can inhibit the nuclear localization of the fusion protein SV8PK9. The lack of nuclear localization of these fusion proteins is not due to their large size, because a fusion protein containing the entire ICP8 sequence fused to PK was able to localize to the nucleus (SVPK7; Fig. 5C). The finding that residues 585 to 1082 were common to the three fusion proteins which failed to localize to the nucleus suggested that this region of ICP8 can inhibit nuclear localization. This finding is consistent with previous results in which deletions within this region have little effect on ICP8 nuclear localization (Fig. 2 and 3). This region of ICP8 is distant from the 28 residues of the nuclear localization signal of ICP8 in the linear polypeptide chain of the fusion proteins because of the insertion of PK.

The addition of certain amino-terminal sequences of ICP8 could lead to an apparent defect in nuclear localization because the protein was cleaved, separating the nuclear localization signal from the PK moiety. To address this possibility, we performed immunofluorescence studies with monoclonal antibody $10-E 3$, which recognizes the carboxyterminal 28 residues of ICP8, including the nuclear localization signal. Subcellular patterns of localization were similar to those shown in Fig. 6 (results not shown). Western immunoblot analysis with monoclonal antibody 10-E3 of the fusion proteins expressed by SV8PK11 and SV8PK12 showed only full-size proteins of the expected sizes of 130 and $124 \mathrm{kDa}$ (results not shown). Thus, the nuclear localization signal is localized in the cytoplasm in the intact, full-length fusion protein. We conclude that the function of a nuclear localization signal can be altered by distant protein sequences on the polypeptide chain. 
Plasmid Proteinencoded

SV8PK1

SV8PK2

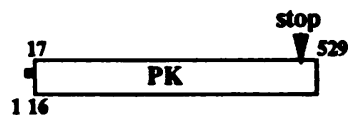

SV8PK3
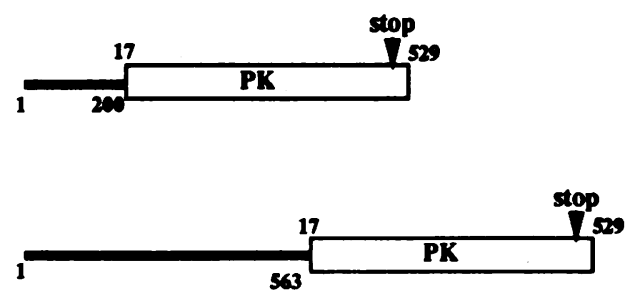

SV8PK4

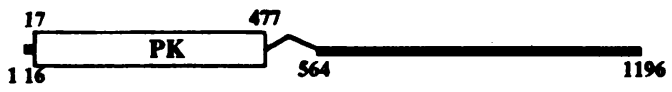

SV8PK5

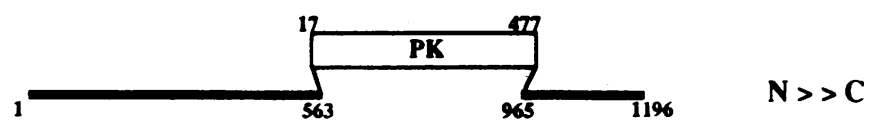

SV8PK6

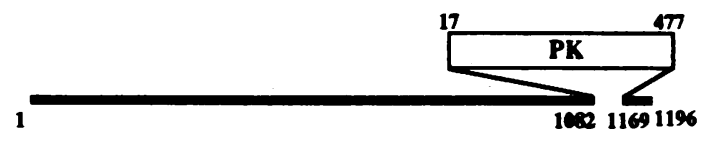

SV8PK8

SV8PK9
$\mathbf{N}$
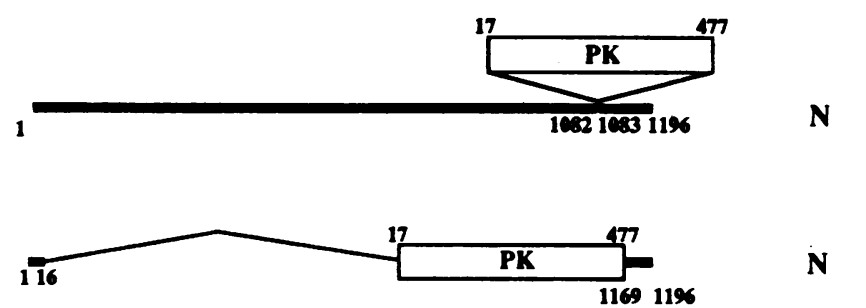

$\mathbf{N}$

$\mathbf{N}$
$\mathbf{N}$

C

C

C

$\mathbf{N} \geq \mathbf{C}$

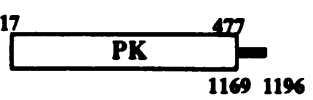

FIG. 4. Structures and subcellular localization of ICP8-PK fusion proteins. Open bars represent the PK sequence, with amino acid residue numbers indicated above; black bars represent the ICP8 sequence, with ICP8 amino acid residue numbers indicated below. N, nucleus; C, cytoplasm.

\section{DISCUSSION}

We have examined ICP8 localization in Vero cells transfected with mutant ICP8 gene plasmids and have shown that the nuclear localization of ICP8 is affected by several regions of the molecule. These regions include the carboxyl-terminal 28 residues and several regions in the amino-terminal half of the molecule. By analysis of deletion mutants and fusion proteins, we showed that the carboxyl-terminal 28 residues of ICP8 are not only absolutely required for its own nuclear localization but also sufficient to direct the normally cytoplasmic PK into the nucleus.

Nuclear localization signals are often, but not exclusively, defined by a stretch of basic amino acid residues $(3,19,39)$. We analyzed the amino acid sequence of ICP8 and found regions of basic residues along the entire length of the ICP8 molecule. We found limited sequence similarity between the carboxyl-terminal 28 residues of ICP8 and the SV40 T-antigen nuclear localization signal sequences (basic residues are underlined, and acidic residues have dots on top):

\section{SV40 $\mathrm{T}$ antigen PPKKKKRKV \\ ICP8 GRKRAFHGDD்PFGEGPPD்KKGD்LTLD்L 1196}

Analysis of the carboxyl-terminal 28 residues of ICP8 indicates that the net charge of this stretch of amino acids is zero, although the first seven positions contain four basic residues. Preliminary data indicate that the first 16 of the 28 amino acids are not sufficient to direct PK into the nucleus (12). These results suggest that the sequences required for ICP8 nuclear localization are more complex than those of the SV40 $\mathrm{T}$ antigen. However, we do not know whether this nuclear localization signal of ICP8 consists of a continuous series of residues or of two discontinuous regions separated 

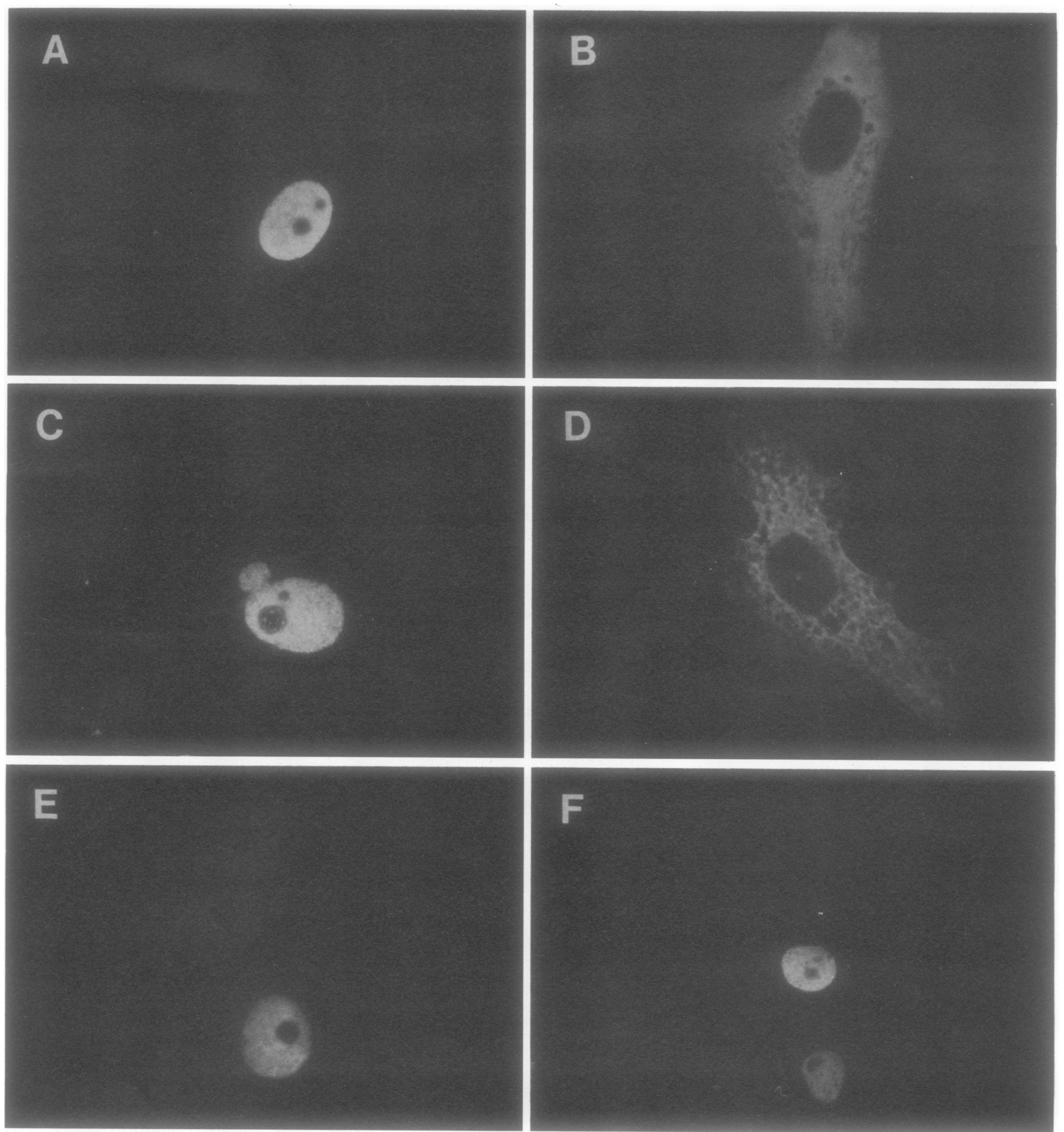

FIG. 5. Localization of chimeric ICP8-PK proteins in Vero cells. The conditions for transfection and immunofluorescence were the same as for Fig. 3 except that anti-PK serum was used. (A) PK10-transfected cells; (B) PK12-transfected cells; (C) pSV8PK7-transfected cells; (D) pSV8PK3-transfected cells; (E) pSV8PK5-transfected cells; (F) pSV8PK9-transfected cells.

by an intervening sequence. Further studies are needed to determine the minimal sequence that will direct PK into the nucleus.

Some of the amino-terminal deletion mutant products (such as SVdXhoI ICP8) and the fusion proteins (such as SV8PK11) contain the carboxyl-terminal 28 residues but fail to localize to the nucleus. Several possible explanations exist for this observation. One possibility is that there are multiple nuclear localization signals in the ICP8 molecule. One contained in the carboxyl-terminal 28 residues of ICP8 is necessary and sufficient to serve as a nuclear localization signal, while the other signals are minor ones. These minor 


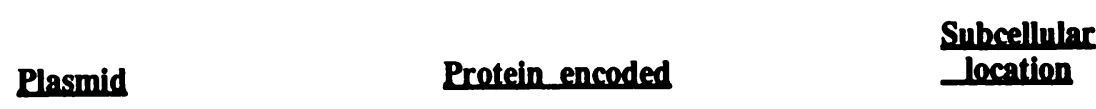

SVPK8

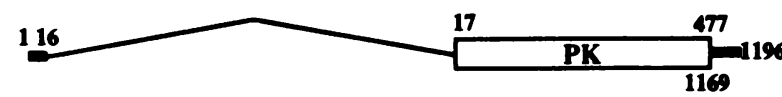

$\mathbf{N}$

SV8PK11

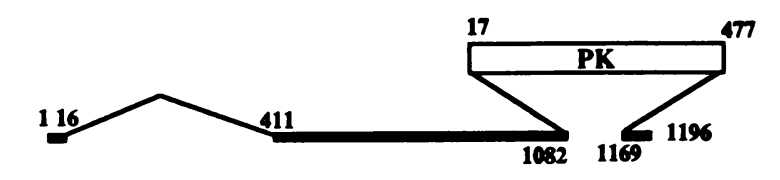

C
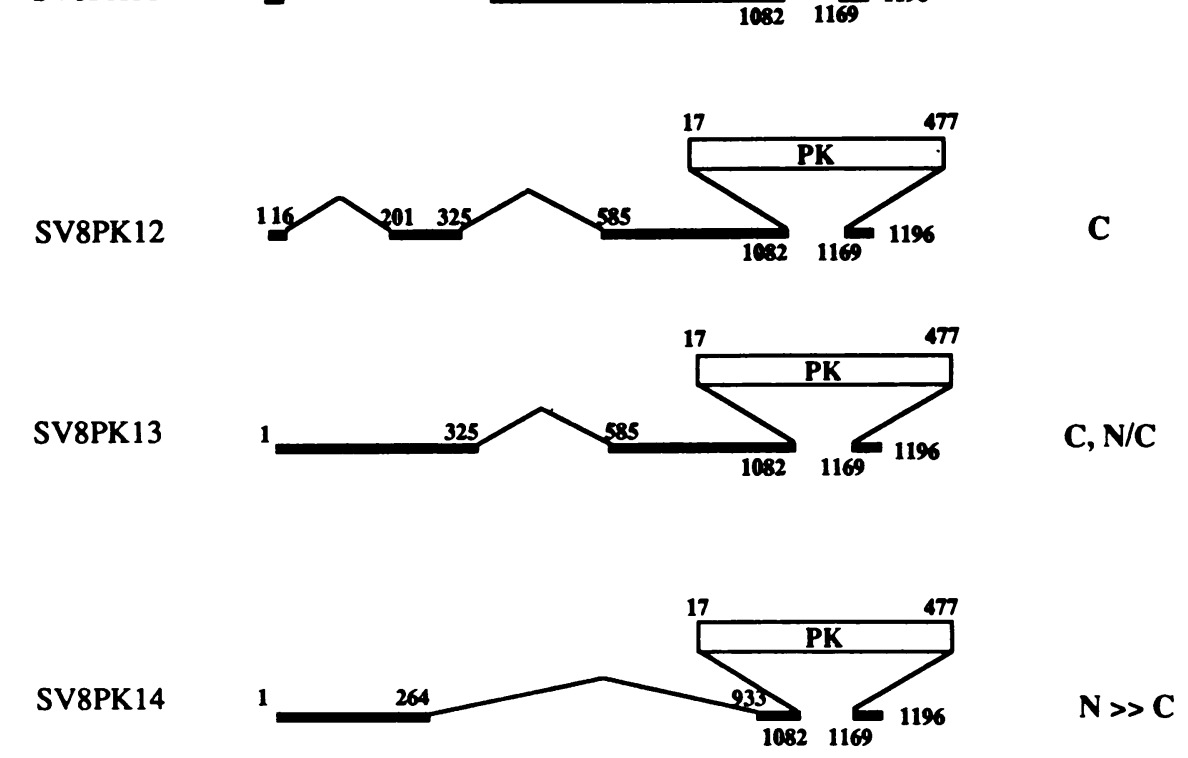

FIG. 6. Structures and subcellular localization of ICP8-PK fusion proteins. Open bars represent the PK sequence, with the positions of amino acids indicated above; black bars represent the ICP8 sequence. $\mathrm{N}$, nucleus; $\mathrm{C}$, cytoplasm.

nuclear localization signals may not have the same ability to serve as a nuclear localization signal for PK as do the signals within the carboxyl-terminal 28 amino acids, but they are important accessory signals determining the nuclear localization of ICP8. Therefore, it is possible that the aminoterminal segment of ICP8 contains multiple minor signals which independently are not sufficient to target PK into the nucleus but which can contribute the accumulation of ICP8 in the nucleus in combination with the carboxyl-terminal 28 residues.

Another possibility is that the conformation of ICP8 is important for its nuclear localization. In this case, deletion of certain regions and even some point mutations might alter the conformation of the molecule and affect the nuclear localization function of the carboxyl-terminal 28 residues. Some work on nuclear proteins has indicated that nuclear localization signals are usually located at ends of molecules $(13,25,43,48)$, where they are exposed on the surface of the proteins in order to exert their function. It has been reported that the SV40 T-antigen nuclear localization signal can function when fused to either the carboxyl or amino terminus of heterologous proteins such as PK but not when inserted at some internal sites within PK (40). Similar effects may be seen with ICP8. It is conceivable that the carboxylterminal segment of the ICP8 molecules is exposed on the protein surface, while the amino terminus is involved in folding the molecules properly for their nuclear localization. Therefore, when regions within the amino terminus of ICP8 are deleted, the ICP8 molecules may not fold properly and the last 28 residues may no longer be exposed on the surface of the protein, where they function. Alternatively, the deletion of sequences adjacent to the carboxyl-terminal 28 residues could alter the conformation of ICP8 more dramatically than does deletion of sequences distant from them. However, removal of residues adjacent or close to the carboxylterminal 28 residues of ICP8 has little effect on nuclear localization (SVd105, SVdPst, and SVd111). Similar results are also observed when some regions of ICP8 are replaced with PK (SV8PK5 and SV8PK6). Furthermore, insertion of more than 500 amino acids of PK between residues 1082 and 1083 of ICP8, immediately adjacent to its nuclear localization signal, has no effect on its nuclear localization (SV8 PK7). These results would argue that a conformational change is the major factor responsible for the lack of nuclear localization for some mutant ICP8 and fusion proteins containing the intact carboxyl-terminal 28 residues.

A third possibility is that the carboxyl-terminal 28 residues serve as the only nuclear localization signal for ICP8, but residues 585 to 1082 interfere in some way with the function of the carboxyl-terminal signal unless the amino-terminal half of ICP8 is present to stabilize the structure. This hypothesis is consistent with our data from deletion mutant and fusion protein analyses. Deletions and PK insertions between residues 585 and 1082 had no effect on nuclear localization. This result is further supported by the subcellular locations of the fusion proteins expressed from 

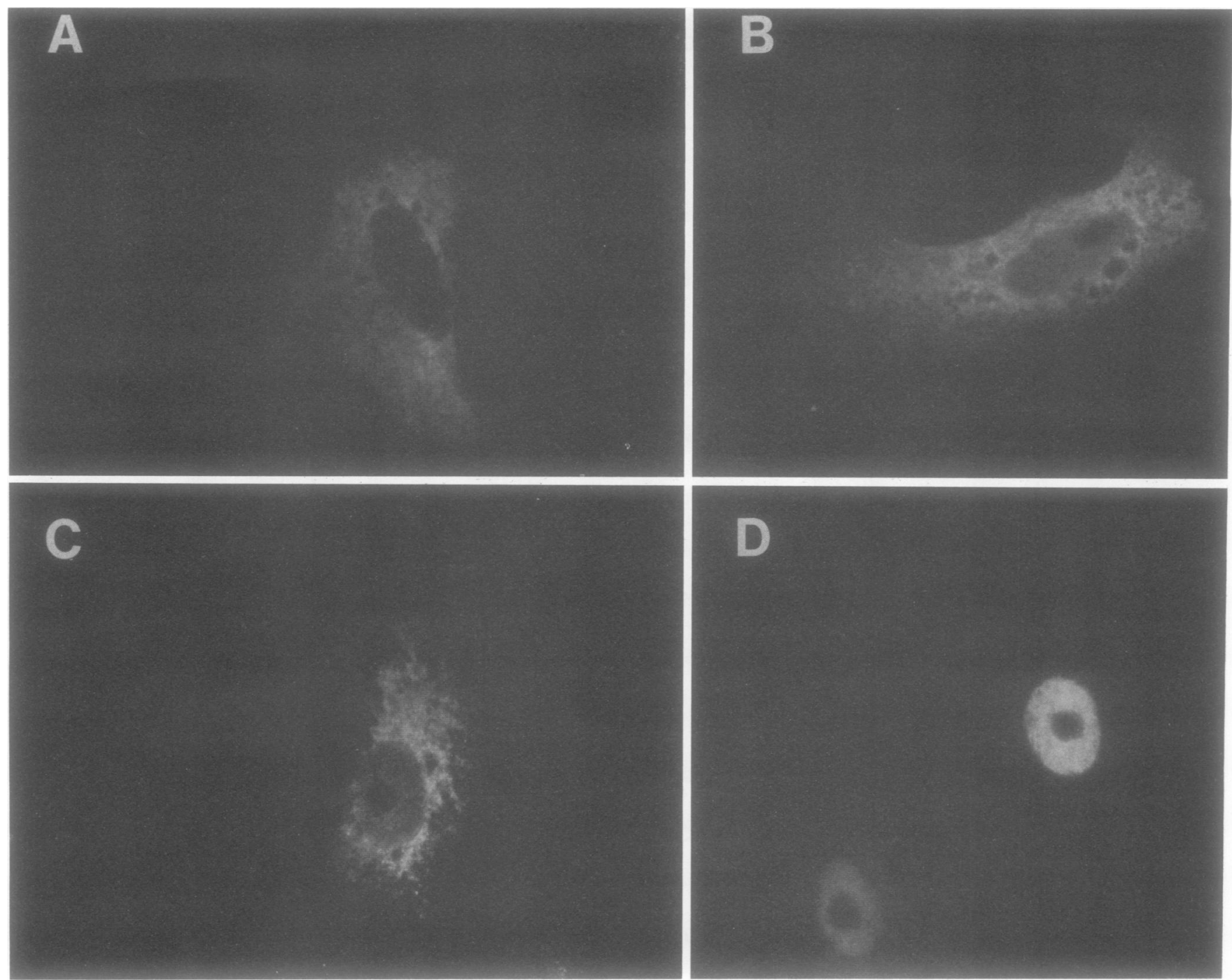

FIG. 7. Localization of chimeric ICP8-PK proteins in Vero cells. The conditions for transfection and immunofluorescence were as described in the legend to Fig. 5. (A) pSV8PK11-transfected cells; (B) pSV8PK12-transfected cells; (C) pSV8PK13-transfected cells; (D) pSV8PK14-transfected cells.

pSV8PK11 and pSV8PK12. Both fusion proteins were derived from SV8PK8, which was entirely localized in the nucleus, but when additional ICP8 sequences from residues 585 to 1082 were added back to generate the fusion proteins (SV8PK11 and SV8PK12), they lost their ability to localize to the nucleus. As residues 585 to 1082 were added, less accumulation of ICP8 in the nucleus was observed. When the entire ICP8 sequence was added back to SV8PK9, the amino terminus of ICP8 could stabilize the structure and the fusion protein was localized in the nucleus (SV8PK7).

We have demonstrated that sequences distant from a nuclear localization signal can affect its ability to function by using a PK cassette fused to different portions of the ICP8 protein. Thus, the signals targeting proteins to the nucleus may be more complicated than a simple linear sequence and the protein context immediately surrounding the signal. Protein folding or inhibitory sequences may also influence nuclear localization of proteins.

\section{ACKNOWLEDGMENTS}

We are grateful to Steve Rice, Leslie Schiff, and Tricia Jandris for help in generating antiserum against PK. We thank Steve Rice and Leslie Schiff for critically reading the manuscript and suggesting many improvements.

This research was supported by Public Health Service grant CA 26345 .

\section{REFERENCES}

1. Bayliss, G. J., H. S. Marsden, and J. Hay. 1975. Herpes simplex virus proteins: DNA-binding proteins in infected cells and in the virus structure. Virology 68:124-134.

2. Breeuwer, M., and D. S. Goldfarb. 1990. Facilitated nuclear transport of histone $\mathrm{H} 1$ and other small nucleophilic proteins. Cell 60:999-1008.

3. Burglin, T. R., and E. M. De Robertis. 1987. The nuclear migration signal of Xenopus laevis nucleoplasmin. EMBO J. 6:2617-2625.

4. Challberg, M. D. 1986. A method for identifying the viral genes required for herpesvirus DNA replication. Proc. Natl. Acad. Sci. USA 83:9094-9098. 
5. Conley, A. J., D. M. Knipe, P. C. Jones, and B. Roizman. 1981. Molecular genetics of herpes simplex virus. VII. Characterization of a temperature-sensitive mutant produced by in vitro mutagenesis and defective in DNA synthesis and accumulation of polypeptides. J. Virol. 37:413-428.

6. Davey, J., N. J. Dimmock, and A. Colman. 1985. Identification of the sequence responsible for the nuclear accumulation of the influenza virus nucleoprotein in xenopus oocytes. Cell 40:667675.

7. Dingwall, C., and R. A. Laskey. 1986. Protein import into the nucleus. Annu. Rev. Cell Biol. 2:367-390.

8. Dingwall, C., J. Robbins, S. M. Dilworth, B. Roberts, and W. D. Richardson. 1988. The nucleoplasmin nuclear location sequence is larger and more complex than that of SV40 large T antigen. J. Cell Biol. 107:841-849.

9. Gao, M., J. Bouchey, K. Curtin, and D. M. Knipe. 1988. Genetic identification of a portion of the herpes simplex virus ICP8 protein required for DNA binding. Virology 163:319-329.

10. Gao, M., and D. M. Knipe. 1989. Genetic evidence for multiple nuclear function of the herpes simplex virus ICP8 DNA-binding protein. J. Virol. 63:5258-5267.

11. Gao, M., and D. M. Knipe. 1991. Potential role for herpes simplex virus DNA replication protein in stimulation of late gene expression. J. Virol. 65:2666-2675.

12. Gao, M., and D. M. Knipe. Unpublished data.

13. Gharakhanian, E., J. Takahashi, and H. Kasamatsu. 1987. The carboxy 35 amino acids of SV40 VP3 are essential for its nuclear accumulation. Virology 157:440-448.

14. Godowski, P. J., and D. M. Knipe. 1986. Transcriptional control of herpesvirus gene expression: gene functions required for positive and negative regulation. Proc. Natl. Acad. Sci. USA 83:256-260.

15. Greenspan, D., P. Palese, and M. Krystal. 1988. Two nuclear location signals in the influenza virus NS1 nonstructural protein. J. Virol. 62:3020-3026.

16. Guiochon-Mantel, A., H. Loosfelt, P. Lescop, S. Sar, M. Atger, M. Perrot-Applanat, and E. Milgrom. 1989. Mechanisms of nuclear localization of the progesterone receptor: evidence for interaction between monomers. Cell 57:1147-1154.

17. Hall, M. N., L. Hereford, and I. Herskowitz. 1984. Targeting of $E$. coli $\beta$-galactosidase to the nucleus in yeast. Cell 36:10571065.

18. Holland, L. E., R. M. Sandri-Goldin, A. L. Goldin, J. C. Glorioso, and M. Levine. 1984. Transcriptional and genetical analysis of the herpes simplex virus type 1 genome: coordinates 0.29 to 0.45 . J. Virol. 49:947-959.

19. Kalderon, D., W. D. Richardson, A. F. Markham, and A. E. Smith. 1984. Sequence requirements for nuclear localization of SV40 large T antigen. Nature (London) 311:33-38.

20. Kalderon, D., B. L. Roberts, W. D. Richardson, and A. E. Smith. 1984. A short amino acid sequence able to specify nuclear location. Cell 39:499-509.

21. Knipe, D. M., D. Senechek, S. A. Rice, and J. L. Smith. 1987. Stages in the nuclear association of the herpes simplex virus transcriptional activator protein ICP4. J. Virol. 61:276-284.

22. Landford, R. E., and J. S. Butel. 1984. Construction and characterization of an SV40 mutant defective in nuclear transport of $\mathrm{T}$ antigen. Cell 37:801-813.

23. Lee, C. K., and D. M. Knipe. 1985. Immunoassay for the DNA-binding activities of herpes simplex virus protein ICP8. J. Virol. 54:731-738.

24. Lonberg, N., and W. Gilbert. 1983. Primary structure of chicken muscle pyruvate kinase mRNA. Proc. Natl. Acad. Sci. USA 80:3661-3665.

25. Lyons, R. H., B. Q. Ferguson, and M. Rosenberg. 1987. Pentapeptide nuclear localization signal in adenovirus E1A. Mol. Cell. Biol. 7:2451-2456.

26. Moreland, R. B., G. L. Langevin, R. H. Singer, R. L. Garcea, and L. M. Hereford. 1987. Amino acid sequences that determine the nuclear localization of yeast histone 2B. Mol. Cell. Biol. 7:4048-4057.

27. Moreland, R. B., H. G. Nam, L. M. Hereford, and H. M. Fried.
1985. Identification of a nuclear localization signal of a yeast ribosomal protein. Proc. Natl. Acad. Sci. USA 82:6561-6565.

28. Morin, N., C. Delsert, and D. F. Klessig. 1989. Nuclear localization of the adenovirus DNA-binding protein: requirement for two signals and complementation during viral infection. Mol. Cell. Biol. 9:4372-4380.

29. Nath, S. T., and D. P. Nayak. 1990. Function of two discrete regions is required for nuclear localization of polymerase basic protein 1 of A/WSN/33 influenza virus (H1N1). Mol. Cell. Biol. 10:4139-4145.

30. Nelson, M., and P. Silver. 1989. Context affects nuclear protein localization in Saccharomyces cerevisiae. Mol. Cell. Biol. 9:384-389.

31. Newmeyer, D. D., and D. J. Forbes. 1988. Nuclear import can be separated into different steps in vitro: nuclear pore binding and translocation. Cell 52:641-653.

32. Orberg, P. K., and P. A. Schaffer. 1987. Expression of herpes simplex virus type 1 major DNA-binding protein, ICP8, in transformed cell lines: Complementation of deletion mutants and inhibition of wild-type virus. J. Virol. 61:1136-1146.

33. Picard, D., and K. R. Yamamoto. 1987. Two signals mediate hormone-dependent nuclear localization of the glucocorticoid receptor. EMBO J. 6:3333-3340.

34. Powell, K. L., E. Littler, and D. J. M. Purifoy. 1981. Nonstructural proteins of herpes simplex virus. II. Major virus-specific DNA-binding protein. J. Virol. 39:894-902.

35. Purify, D. J. M., and K. L. Powell. 1976. DNA-binding proteins induced by herpes simplex virus type 2 in HEp-2 cells. J. Virol. 19:717-731.

36. Quinlan, M. P., L. B. Chen, and D. M. Knipe. 1984. The intranuclear location of a herpes simplex virus DNA-binding protein is determined by the status of viral DNA replication. Cell 36:857-868.

37. Quinlan, M. P., and D. M. Knipe. 1983. Nuclear localization of herpesvirus protein: potential role for the cellular framework. Mol. Cell. Biol. 3:315-324.

38. Richardson, W. D., A. D. Mill, S. M. Dilworth, R. A. Laskey, and C. Dingwell. 1988. Nuclear protein migration involves two steps rapid binding at the nuclear envelope followed by slower translocation through nuclear pores. Cell 52:655-664.

39. Richardson, W. D., B. L. Roberts, and A. E. Smith. 1986. Nuclear location signals in polyoma virus large T. Cell 44:77-85.

40. Roberts, B. L., W. D. Richardson, and A. E. Smith. 1987. The effect of protein context on nuclear location signal function. Cell 50:465-475.

41. Rose, D. S. C., K. Shriver, D. S. Latchman, and N. B. Lathangue. 1986. A filamentous distribution for the herpes simplex virus type 2-encoded major DNA binding protein. J. Gen. Virol. 67:1315-1325.

42. Shaulsky, G., Goldfinger, A. Ben-Ze'ev, and V. Rotter. 1990. Nuclear accumulation of p53 protein is mediated by several nuclear localization signals and plays a role in tumorigenesis. Mol. Cell. Biol. 10:6565-6577.

43. Silver, P., L. Keegan, and M. Ptashne. 1984. Amino terminus of the yeast GAL4 gene product is sufficient for nuclear localization. Proc. Natl. Acad. Sci. USA 81:5951-5955.

44. Smith, A. E., D. Kalderon, B. L. Roberts, W. H. Colledge, M. Edge, P. Gillet, A. Markham, E. Paucha, and W. D. Richardson. 1985. The nuclear location signal. Proc. R. Soc. London Biol. Sci. 226:43-58.

45. Spang, A. E., P. J. Godowski, and D. M. Knipe. 1983. Characterization of herpes simplex virus 2 temperature-sensitive mutants whose lesions map in or near the coding sequences for the major DNA-binding protein. J. Virol. 45:332-342.

46. Stuart, D. I., M. Levine, H. Murirhead, and D. K. Stammers. 1979. Crystal structure of cat muscle pyruvate kinase at a resolution of 2.6 A. J. Mol. Biol. 134:109-142.

47. Villarreal, E., and D. M. Knipe. Unpublished data.

48. Wychowiki, C., D. Benichou, and M. Girard. 1986. A domain of SV40 capsid polypeptide VP1 that specifies migration into the cell nucleus. EMBO J. 5:2569-2576. 